\title{
Étude des paramètres ayant une influence sur le comportement au fluage d'un sable injecté
}

E. DELFOSSE-RIBAY I. DJERAN-MAIGRE

R. CABRILLAC

Laboratoire de Modélisation

Matériaux et Structures (LMFS)

CNRS UMR 7143

Université

de Cergy-Pontoise

5 mail Gay-Lussac 95031 Cergy-Pontoise Cedex estelle_delfosse@yahoo.fr irini.djeran-maigre@iupgc.ucergy.fr

richard.cabrillac@iupgc.ucergy.fr

\section{GOUVENOT}

\section{E. DELFOSSE-RIBAY}

Soletanche Bachy

6. rue de Watford 92005 Nanterre Cedex Daniel.GOUVENOT@ soletanche-bachy.com

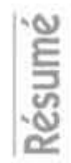

Le comportement au fluage du sable de Fontainebleau injecté par un gel de silice, chimiquement instable est comparé au comportement au fluage du même sable injecté par deux coulis de nouvelle génération chimiquement stables, un coulis de ciment micro-fin et un coulis minéral. Le comportement au fluage est caractérisé par des essais en compression simple et des essais avec confinement réalisés sur des périodes allant de 100 à 200 jours. Les résultats sont analysés en termes de pente de fluage et de charge limite de fluage. L'analyse des essais a mis en évidence que le mode de mise en place du sable joue un rôle important sur les déformations de fluage du sable injecté. Les déformations de fluage sont considérablement réduites par l'application d'une contrainte de confinement. Le traitement d'injection modifie le comportement au fluage du sable : selon la nature du coulis injecté, le sable injecté présente des déformations de fluage plus ou moins importantes. Les pentes de fluage du sable injecté sont proportionnelles aux pentes de fluage du coulis pur. Les caractéristiques au fluage du sable injecté semblent ainsi pouvoir être déduites du comportement au fluage du sable non traité et du comportement au fluage du coulis pur.

Mots-clés : fluage, sable, injection, gel de silice, coulis de ciment, coulis minéral.

\section{Study of parameters affecting the creep behaviour of a grouted sand}

The creep behaviour of the Fontainebleau sand grouted with a chemically unstable silicate grout was compared with the creep behaviour of the same sand grouted with two chemically stable grouts, a micro-fine cement-based grout and a mineral-based grout. Unconfined and confined creep experiments were performed to characterise drained creep behaviour. Tests duration was between 100 and 200 days. Results were compared in terms of creep slope, and creep limit strength. Tests results have shown that the method with which sand was prepared plays an important part in creep strains of grouted sand. Creep strains were considerably reduced by applying a confining stress. The creep behaviour of sands was modified by the grouting treatment: depending on the nature of the grout, the grouted sand has presented more or less large creep strains. The creep slopes of grouted sands were proportional to the creep slopes of pure grouts. Creep characteristics of grouted sand may also be decluced from the creep behaviour of pure sand and the creep behaviour of pure grout.

NDLR: Les discussions sur 


\section{Introduction}

La raréfaction des sols de bonne qualité utilisables pour la construction, le développement des travaux en sous-cuvre ont conduit au développement ces dernières années de nouvelles techniques destinées à améliorer les caractéristiques des sols en place. L'une de ces techniques est l'injection qui consiste à faire pénétrer dans les sols un produit liquide appelé " coulis $»$, qui après durcissement améliore les caractéristiques mécaniques et la perméabilité du sol. Depuis un siècle, la technique de l'injection a fait l'objet de nombreuses évolutions et innovations à tous les niveaux, de la surveillance des chantiers au contrôle qualité final (Gouvenot, 1998). En ce qui concerne les coulis d'injection, le gel de silice, coulis chimique, a été utilisé pendant de nombreuses années. C'est un coulis sujet à la synérèse, donc instable chimiquement. Sa dégradation libère de fortes concentrations de soude et des dérivés organiques. Ces produits entraînés par l'eau constituent un facteur de pollution de la nappe phréatique. Pour cette raison, le gel de silice est maintenant interdit dans certains pays d'Europe et au Japon (Gouvenot, 1987). Pour pallier ces problèmes de pollution, de nouveaux coulis ont été développés comme les coulis de ciment micro-fin ou les coulis à base minérale qui sont composés aprés durcissement d'éléments stables tels que les cristaux de silicates de calcium hydratés (CSH). Cette stabilité chimique a permis de réduire considérablement les effets néfastes de l'injection. Outre la stabilité chimique, une des préoccupations actuelles est la durabilité mécanique des sols traités, c'est-à-dire la stabilité de leurs propriétés mécaniques dans le temps. Cette étude s'est intéressée en particuliers à un aspect de la durabilité dans le temps : le comportement au fluage. En effet, le fluage est un phénomène important qui ne doit pas être négligé car il peut conduire à la rupture des matériaux pour des niveaux de contraintes parfois beaucoup plus faibles que la résistance instantanée. En ingénierie géotechnique, les caractéristiques de déformations différées peuvent conduire à de graves désordres comme les tassements ou les glissements de terrains. Une meilleure connaissance du comportement à long terme des sols injectés permettra de ne plus utiliser l'injection uniquement comme traitement provisoire, comme c'est le cas pour le sable injecté au gel de silice, mais également d'envisager son utilisation en tant que traitement définitif. De nombreuses études traitent du fluage du sable injecté par du gel de silice (Borden et al., 1982 ; Krizek et al., 1982 ; Luong et al., 1977 ; Mollamahmutoglu, 1992) mais peu d'études sont disponibles sur le comportement à long terme du sable injecté par des coulis de nouvelle génération (Tailliez, 1998). Cette étude a été menée afin de comparer le comportement au fluage d'un sable injecté par les nouveaux coulis avec celui du même sable injecté par un gel de silice classique. Elle a pour objet de mettre en évidence l'influence du traitement par injection sur le comportement au fluage du sable, et notamment Y'influence de la nature du coulis. Pour cela différents essais de fluage, en compression simple ou avec un confinement, ont été réalisés sur des durées allant de 3 à 6 mois. Outre les effets du traitement d'injection et de la nature du coulis, d'autres facteurs, tels que le mode de mise en place du sable, le type de chargement ou la présence d'un confinement ont été également analysés.

\section{Caractéristiques et préparation des matériaux} 2.1

\section{Caractéristiques des matériaux}

\section{6 \\ Matrice granulaire}

Le sable de Fontainebleau (SF) NE34 a été utilisé pour l'ensemble des essais réalisés. Il s'agit d'un sable fin, siliceux, à grains très résistants et anguleux. Les caractéristiques du sable sont les suivantes:

- masse volumique des grains : $2,64 \mathrm{~g} / \mathrm{cm}^{3}$;

- diamètre des grains à $10 \%$ de passant : $D_{10}=0,14$;

- coefficient d'uniformité : 1,6;

- masse volumique minimale : $1,39 \mathrm{~g} / \mathrm{cm}^{3}$;

- masse volumique maximale : $1,75 \mathrm{~g} / \mathrm{cm}^{3}$.

\section{2:15:}

\section{Coulis d'injection}

Trois types de coulis ont été utilisés.

- un gel de silicate de soude, composé de silicate de sodium, d'eau et d'un durcisseur organique $(600 \mathrm{C}$ de chez Rhône Poulenc). Le rapport pondéral ( $\mathrm{Rp}=$ $\mathrm{SiO} / \mathrm{Na}, \mathrm{O})$ du gel utilisé vaut 3,3 . La répartition en poids de chacun des constituants est $50 \%$ de silicate de sodium, $42 \%$ d'eau et $8 \%$ de durcisseur. Le temps de prise du coulis est de 24 minutes lorsque la température de l'air est de $24^{\circ} \mathrm{C}$;

- un coulis de ciment micro-fin (Microsol breveté par la société Soletanche Bachy). Il s'agit d'une suspension de grains de ciment dans l'eau. Les grains ont un diamètre inférieur à $12 \mu \mathrm{m}$;

- un coulis minéral (Silacsol breveté par la société Soletanche Bachy). C'est un coulis chimique. Il s'agit d'une liqueur de silice réglée pour réagir directement sur les milieux contenant du calcium. La réaction dans le milieu injecté conduit à la précipitation de cristaux de silicates de calcium hydratés.

\section{Coulis pur stabilisé}

La réalisation d'essais mécaniques sur coulis purs, après prise, implique de disposer de matériaux stables pour que les échantillons soient homogènes. Le coulis de ciment micro-fin et le coulis minéral sont des coulis instables dans la mesure où ils présentent un phénomène de ressuée, formation d'une couche d'eau à la surface du coulis lorsqu'ils sont laissés au repos, supérieure à $5 \%$. Ce phénomène est souvent simultané avec la sédimentation des grains constituant le coulis. L'addition de bentonite est une méthode classique pour éviter la sédimentation du coulis car la bentonite hydratée maintient en suspension les grains consti- 
tuant le coulis. Ainsi, de la bentonite en faible quantité, environ $1 \%$ du volume du coulis, a été ajoutée à la composition initiale des coulis de ciment et coulis minéral. Avec la bentonite, le phénomène de ressuée est inférieur à $5 \%$, les coulis sont donc considérés comme stables. L'effet de l'ajout de bentonite est difficile à estimer puisque le coulis pur sans la bentonite n'est pas stable et qu'il n'a pas été possible de réaliser d'essais sur ce matériau afin de comparer ses caractéristiques mécaniques avec celles du coulis stabilisé. Selon Cambefort (1967), si la bentonite permet d'obtenir un coulis homogène, le temps de prise est retardé et la résistance mécanique est diminuée. Cependant, pour les coulis étudiés, la quantité de bentonite ajoutée est très faible et il peut ètre supposé que l'effet de la bentonite est également faible.

En ce qui concerne le gel de silice, il est soumis au phénomène de synérèse qui se traduit par une contraction du coulis. Il est chimiquement instable et ne peut pas être stabilisé.

\section{2}

\section{Préparation des échantillons de sable injecté}

Les différentes étapes de la préparation des échantillons sont les suivantes:

- Le sable est mis en place dans des colonnes cylindriques en Plexiglas de $80 \mathrm{~mm}$ de diamètre. Deux méthodes de mise en place du sable ont été utilisées :

- la densification manuelle, quí consiste à introduire le sable progressivement et à le densifier en tapant fortement et régulièrement sur le pourtour de la colonne. L'indice de densité obtenu par cette méthode est de 0,90

- la pluviation (Cresswell et al., 1999) qui consiste à faire tomber le sable à débit et hauteur de chute constants. L'indice de densité ainsi obtenu est de 0,84.

- Les colonnes de sable sont saturées en faisant passer un certain volume d'eau dans la colonne. L'eau entre par le bas de la colonne et monte progressivement à débit constant égal à $3 \mathrm{~cm}^{3} / \mathrm{s}$. A la fin de cette phase, le degré de saturation est de $94 \%$.

- Les colonnes sont ensuite injectées en faisant entrer de bas en haut un certain volume de coulis à débit constant identique à celui de la saturation. Le coulis est agité pendant toute la phase de l'injection pour éviter la décantation de ses grains. L'injection est arrêtée lorsqu'une quantité de coulis, correspondant à environ $120 \%$ du volume des vides, est passée à travers l'échantillon.

- Les colonnes sont ensuite conservées en milieu humide 28 jours pour les colonnes injectées au coulis de ciment micro-fin et au coulis minéral et 7 jours pour celles injectées au gel de silice. Pour ces dernières, plusieurs études (Christopher et al., 1989 ; Krizek et al., 1982; Littlejohn et al., 1994) ont en effet montré que la résistance maximale en compression simple est atteinte en 7 jours. Les colonnes sont alors sciées afin d'obtenir les dimensions requises pour le dispositif expérimental, soit un diamètre de $80 \mathrm{~mm}$ et une hauteur de $160 \mathrm{~mm}$ pour les essais réalisés en compression simple, et $54 \mathrm{~mm}$ de diamètre et $108 \mathrm{~mm}$ de haut pour les essais réalisés en cellule triaxiale. Pour obtenir le diamètre de $54 \mathrm{~mm}$, les échantillons sont carottés. Le carottage est susceptible d'avoir provoqué des perturbations sur les caractéristiques mécaniques du matériau. Cependant il a été porté une grande attention sur l'aspect de chaque échantillon, parallélisme des faces, fissures non visibles. En outre, des résistances en compression simple réalisées sur des échantillons carottés et non carottés ont donné des résultats très proches, ce qui tend à montrer que l'effet du carottage a été faible. Une colonne fabriquée par pluviation (hauteur $1150 \mathrm{~mm}$ ) donne 5 échantillons et une colonne fabriquée par densification manuelle (hauteur $750 \mathrm{~mm}$ ) donne 3 échantillons (Fig. 1). Les échantillons situés aux extrémités des colonnes sont utilisés pour les résistances en compression simple et les échantillons intermédiaires sont utilisés pour les essais de fluage.

- Comme il a été observé une décroissance linéaire de la résistance en compression simple entre les échantillons placés à l'entrée de la colonne (près du point d'injection) et les échantillons les plus éloignés du point d'injection, des mesures de résistances en compression simple sont réalisées sur les échantillons situés aux extrémités de chaque colonne. L'application d'une régression linéaire entre les résistances de ces deux échantillons permet d'estimer la résistance en compression simple des échantillons intermédiaires, utilisés pour les essais de fluage. L'erreur sur cette évaluation est inférieure à 10\% (Ribay, 2001), Les essais de résistance en compression simple sont réalisés sous une vitesse de chargement de $20 \mathrm{~mm}$ par minute selon les recommandations de l'AFTES 1988. Cette vitesse de chargement est rapide mais est utilisée uniquement pour la détermination des résistances en compression simple (Rc). Ces résistances servent de référence pour la détermination du taux de chargement ou des incréments de chargement pour les essais de fluage. Si la vitesse de chargement pour les résistances en compression simple avait été plus faible, les taux de chargement, pour un même niveau de contrainte appliqué aux échantillons lors des essais de fluage, auraient été légèrement supérieurs à ceux donnés dans l'article, et ce d'autant plus que le matériau est sensible à la vitesse de chargement (c'est-à-dire d'avantage pour le sable injecté au gel de silice que pour le sable injecté par les deux autres coulis).

\section{3}

\section{Programme expérimental}

Le fluage est le phénomène physique quil correspond à l'évolution dans le temps de la déformation à contrainte et à température constantes. Tous les essais ont été réalisés en condition drainée sur des échantillons saturés de sorte que ce soit la contrainte effective appliquée au sol qui reste constante.

\section{1}

\section{Essai de fluage en compression simple}

\section{$344_{1}$ \\ Dispositif expérimental}

Les essais de fluage en compression simple ont été réalisés sur des bâtis de type odométrique modifiés afin de recevoir une cellule cylindrique de $180 \mathrm{~mm}$ de haut et de $130 \mathrm{~mm}$ de diamètre. L'échantillon ayant un 


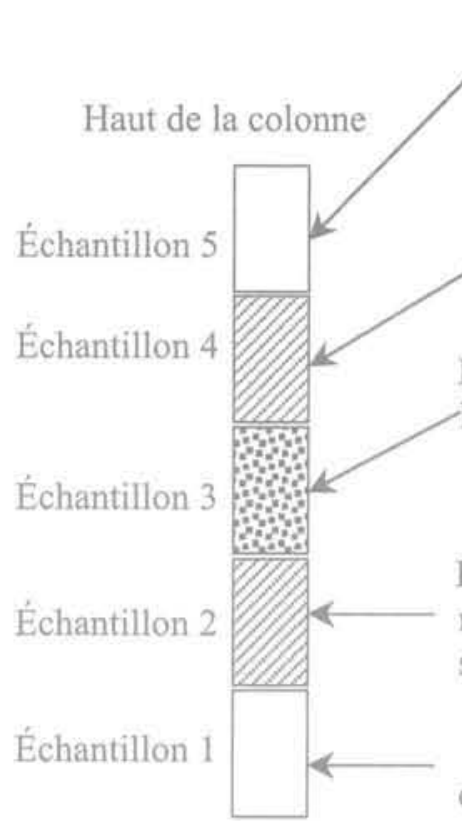

Bas de la colonne
Échantillon éliminé à

cause des effets de bords

Échantillons utilisés pour les résistances en compression simple

Échantillons utilisés pour les essais de fluage

Échantillons utilisés pour les

résistances en compression simple

Échantillons éliminés à cause des effets de bords

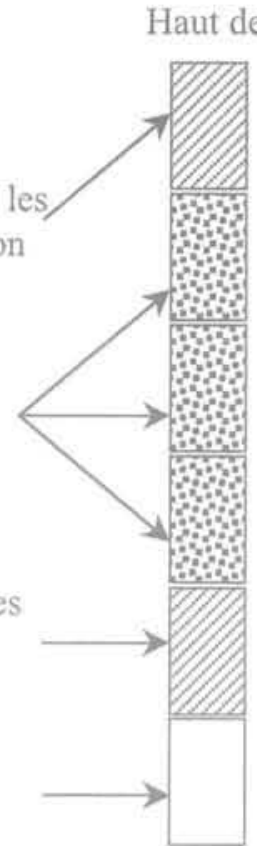

Bas de la colonne

Colonne fabriquée par densification manuelle Hauteur $750 \mathrm{~mm}$

Colonne fabriquée par pluviation

Hauteur $1150 \mathrm{~mm}$

FIG. 1 Schéma de découpe des colonnes.

Schematic representation of the column cutting.

diamètre de $80 \mathrm{~mm}$ il est libre de se déformer latéralement. Seules les déformations axiales sont mesurées avec un comparateur précis au micromètre (Fig. 2). Dans la cellule, huit jours avant le début de l'essai, l'échantillon est placé dans l'eau pour assurer sa saturation. Une fois l'essai de fluage commencé, il reste en contact direct avec l'eau afin de rester saturé pendant toute la durée de l'essai. Le drainage est possible par les faces latérales de l'échantillon. La figure 3 présente une photographie du dispositif.

\subsection{2}

\section{Procédure expérimentale}

L'échantillon est soumis à un chargement axial. La charge (q) appliquee est définie en pourcentage de la résistance en compression simple (Rc) de l'échantillon sous une vitesse de $20 \mathrm{~mm}$ par minute, $\mathrm{q}=0,10 \mathrm{Rc}$ par exemple. La vitesse de chargement moyenne pour les essais de fluage est inférieure à $0,03 \mathrm{~mm} / \mathrm{min}$ (soit $0,02 \% / \mathrm{min})$.

Deux types d'essais de fluage en compression simple ont été réalisés:

- essais de longue durée sur 100 jours, qui consistent à maintenir un chargement constant pendant une durée de 100 jours si la rupture n'intervient pas avant conformément aux recommandations ASTM D 5202-91 (Tableau I) ;

- essais par incréments de courte durée, qui consistent à appliquer un chargement (q), qui constitue le premier increment. Cette charge est maintenue un temps $t$, au bout duquel une nouvelle charge q est appliquée. C'est le second incrément. L'échantillon est alors soumis à une charge totale égale à 2 q qui est maintenue pendant le temps t. Le chargement augmente ainsi jusqu'à la rupture de l'échantillon. La durée des incréments est de 15 minutes ou de 50 minutes selon les essais (Tableau II),

\section{2}

\section{Essai de fluage avec confinement}

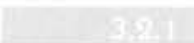 \\ Dispositif expérimental}

Les essais de fluage avec confinement sont réalisés sur un système triaxial. La cellule triaxiale utilisée nécessite des échantillons de $54 \mathrm{~mm}$ de diamètre et de $108 \mathrm{~mm}$ de hauteur. Pour isoler l'échantillon du liquide de confinement (glycérine), deux membranes, une en latex l'autre en Néoprène ont été utilisées afin d'éviter les problèmes de fuite et de limiter le phénomène d'osmose entre le liquide de confinement et l'eau contenue à l'intérieur de l'échantillon. Avant l'essai de fluage, une contre-pression de 0,5 MPa est appliquée aux échantillons pour assurer leur saturation. Celle-ci est vérifiée en condition non drainée, en augmentant légèrement la pression de cellule, et en vérifiant l'augmentation induite de la pression interstitielle. Au cours de l'essai de fluage à proprement dit, le drainage de l'échantillon est possible par les faces supérieure et inférieure de l'échantillon.

Le chargement de l'échantillon est assuré par un piston entraîné par un moteur qui tourne à vitesse constante. La force est mesurée à la fois par un anneau 


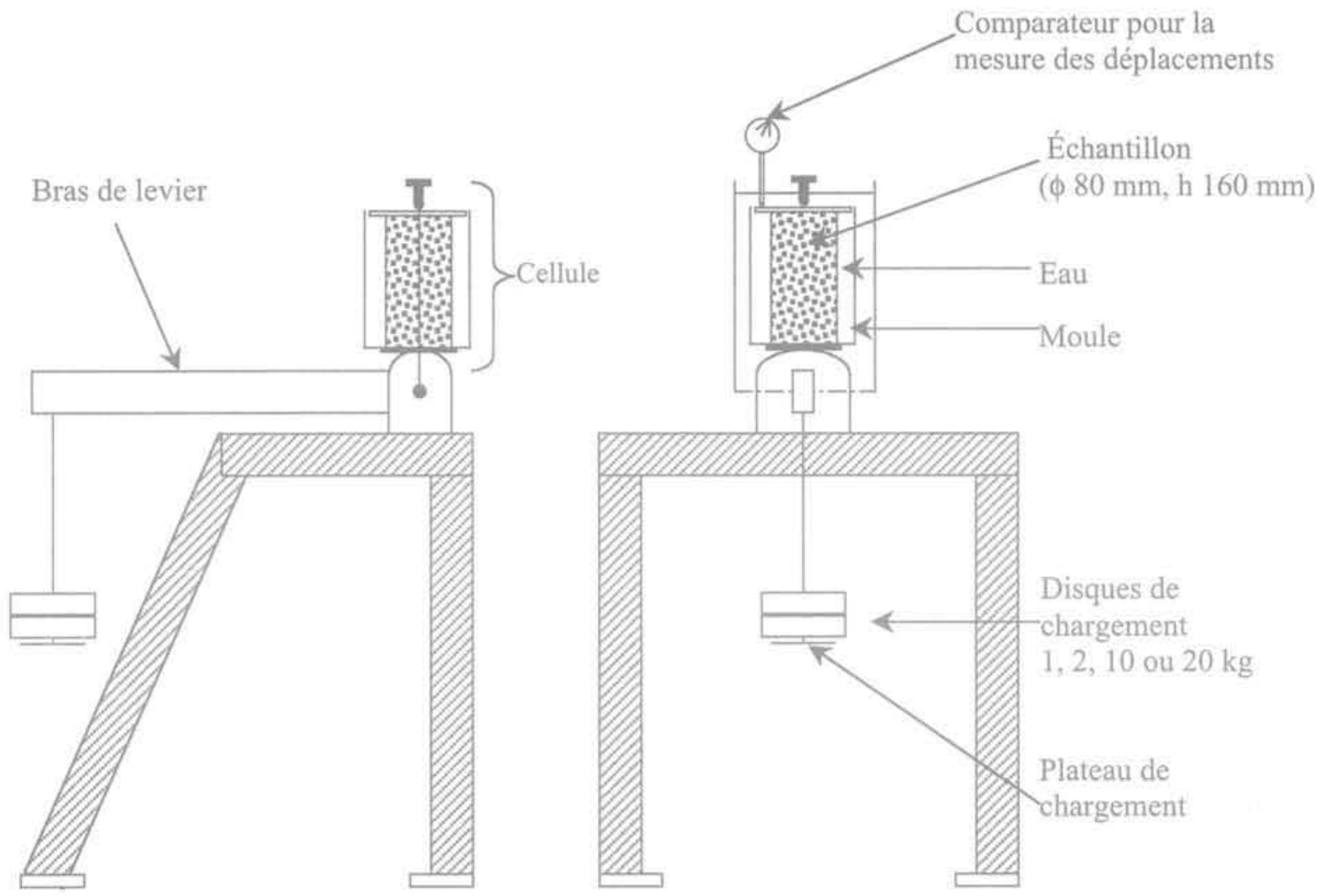

FIG. 2 Schéma du bâti de fluage pour les essais de fluage en compression simple.

Schematic representation of the creep apparatus for unconfined compression creep.

dynamométrique et par un capteur de force. Le système triaxial est équipé d'une jupe tournante, anneau métallique placé autour du piston qui tourne en permanence et permet de limiter les frottements entre le piston et la cellule (Fig. 4). La cellule est également équipée d'un système qui permet d'ajuster automatiquement la force appliquée à l'échantillon si celle-ci varie au cours de l'essai. Les déplacements sont mesurés par un comparateur précis au micromètre et par un LVDT (Linear Variable Differential Transducer). La pression de la cellule et la contre-pression sont contrôlées par des manomètres. Un système de bacs à mercure permet de maintenir une contrainte de confinement constante autour de l'échantillon.

\section{0}

\section{Procédure expérimentale}

Tous les essais de fluage avec confinement ont été réalisés avec une pression de confinement de 0,1 MPa. La charge appliquée est définie en pourcentage de la résistance en compression simple de l'échantillon. L'échantillon est chargé en appliquant une rampe de déformation constante égale à $0,02 \mathrm{~mm}$ par minute. Cette vitesse assure des conditions de chargement drainées en effet des essais triaxiaux statiques (CD) ont été réalisés à cette vitesse de chargement et aucune surpression interstitielle n'a été enregistrée (Ribay, 2001). Deux types d'essais de fluage en confinement ont été réalisés :

- essais de longue durée sur 100 jours, qui consistent à maintenir le chargement constant pendant une durée de 100 jours si la rupture n'intervient pas avant (Tableau III) ;

- essais de fluage par incréments de longue durée. Chaque incrément de charge est maintenu constant jusqu'à ce que les déformations se stabilisent ou jusqu'à ce que l'échantillon se déforme à vitesse constanté. Ainsi, la durée d'un incrément n'est pas constante. Elle dépend du comportement du matériau. Un incrément peut durer entre 7 et 20 jours. La charge est augmentée progressivement jusqu'à la rupture de l'échantillon. Ces essais par incréments ne sont donc pas des essais rapides. La durée totale d'un essai varie entre 4 et 6 mois (Tableau IV).

La figure 5 résume l'ensemble des essais réalisés ainsi que les matériaux testés pour chacun des essais.

\section{Outils d'analyse}

La pente de fluage et la charge limite de fluage ont été utilisées pour analyser et comparer les résultats des essais de fluage.

\section{1}

\section{Pente de fluage}

La pente de fluage permet d'une part de déterminer la charge limite de fluage et d'autre part de quantifier le comportement visqueux des matériaux sans prendre en 


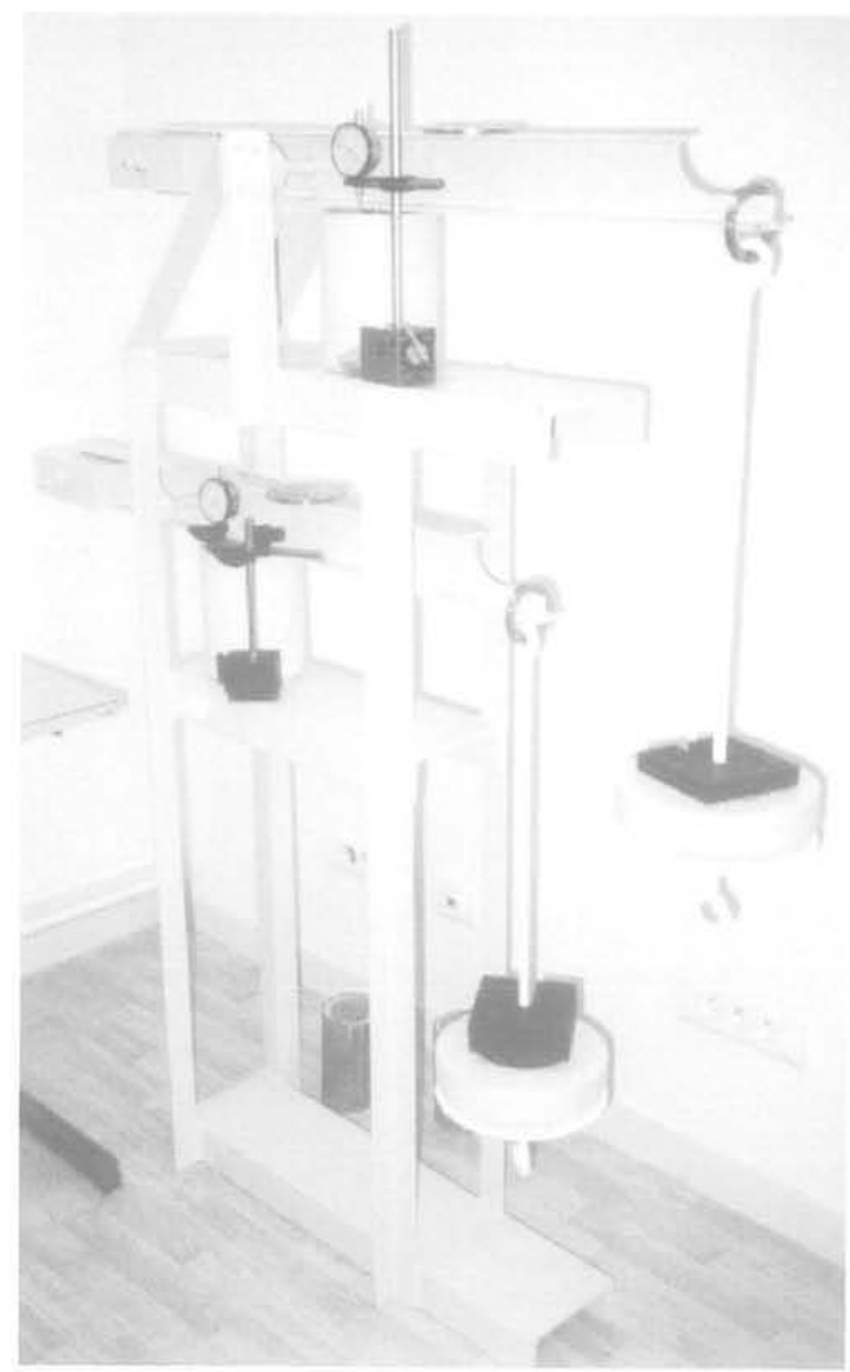

FIG 3 Bâti de fluage pour les essais de fluage en compression simple.

Creep apparatus for unconfined compression creep tests. compte la déformation instantanée. En effet, la valeur de cette dernière peut être perturbée par différents facteurs (état de surface des éprouvettes, mise en contact, etc.). Plusieurs auteurs (Gandais, 1977; Lacour et al., 1978; Murayama et Shibata, 1964; Vialov et Skibitski, 1961; Tan, 1961) ont utilisé la pente de fluage pour analyser leurs résultats mais les méthodes diffèrent quelque peu selon la durée de l'essai et le type de matériau. Pour les matériaux injectés, la méthode la plus adaptée, consiste à calculer les pentes de fluage dans un plan (déformation-temps exprimé en minute). Elles sont données par la pente de la droite de régression linéaire passant par l'ensemble des points dans un intervalle de temps donné, correspondant à $90 \%$ du temps de chargement total de l'échantillon. Ainsi les pentes de fluage sont déterminées:

- entre 10 et 100 jours pour les essais de fluage sur 100 jours en compression simple ou avec confinement; - entre 2 et 15 minutes pour les essais de fluage en compression simple par incréments d'une durée de 15 minutes ;

- entre 5 et 50 minutes pour les essais de fluage en compression simple par incréments d'une durée de 50 minutes :

- entre 1 et 10 jours pour les essais de fluage avec confinement par incréments de longue durée.

\section{2}

\section{Charge limite de fluage}

La charge limite de fluage est aussi connue sous le nom de résistance à long terme. Pour des chargements inférieurs à la charge limite de fluage, le comportement du matériau reste amorti. Pour des chargements supérieurs à la charge limite, le fluage du matériau est de nature non amortie. La rupture par fluage intervient au bout d'un temps fini.

A chaque niveau de chargement correspond une pente de fluage, déterminée selon la méthode décrite

TABLEAU1 Caractéristiques des essais de fluage en compression simple sur 100 jours. Characteristics of the unconfined creep tests performed over a period of 100 days.

\begin{tabular}{l|c|c}
\multicolumn{1}{c|}{$\begin{array}{c}\text { Nature } \\
\text { des échantillons }\end{array}$} & $\begin{array}{c}\text { Méthode } \\
\text { de mise en place } \\
\text { du sable }\end{array}$ & Taux de chargement (.Rc) \\
\hline \multirow{2}{*}{$\mathrm{SF}+$ gel de silice } & $\begin{array}{c}\text { Densification } \\
\text { manuelle }\end{array}$ & $0,025-0,05-0,10(\times 2)-0,25$ \\
\cline { 2 - 3 } & Pluviation & $0,05(\times 3)-0,10(\times 3)$ \\
\hline $\mathrm{SF}+$ coulis de ciment & Pluviation & $0,50-0,60(\times 2)-0,65-0,70(\times 3)$ \\
\hline SF + coutis minéral & Pluviation & $0,35-0,45-0,50-0,60-0,80$ \\
\hline Coulis de ciment pur stabilisé & & $0,50-0,55-0,60-0,70-0,80-0,85$ \\
\hline Coulis minéral pur stabilisé & & $0,30-0,40-0,50-0,60-0,70$
\end{tabular}


TABLEAU II Caractéristiques des essais de fluage en compression simple par incréments.

Characteristics of the incremental unconfined creep tests.

\begin{tabular}{|c|c|c|c|c|}
\hline $\begin{array}{c}\text { Nature } \\
\text { des échantillons }\end{array}$ & $\begin{array}{c}\text { Valeur } \\
\text { de l'incrément }\end{array}$ & $\begin{array}{l}\text { Durée } \\
\text { de l'incrément } \\
\text { (mn) }\end{array}$ & $\begin{array}{l}\text { Nombre } \\
\text { d'incréments } \\
\text { à la rupture }\end{array}$ & $\begin{array}{c}\text { Taux } \\
\text { de chargement } \\
\text { à la rupture }\end{array}$ \\
\hline \multirow{5}{*}{$\begin{array}{l}\mathrm{SF}+\text { gel } \\
\text { de silice }\end{array}$} & $0,05 \mathrm{PC}$ & 15 & 7 & $0,35 \mathrm{Rc}$ \\
\hline & $0,10 \mathrm{Rc}$ & 10 & 5 & $0,50 R C$ \\
\hline & $0,10 \mathrm{Rc}$ & 10 & 5 & $0,50 \mathrm{Re}$ \\
\hline & $0,10 \mathrm{Rc}$ & 50 & 4 & $0.40 \mathrm{Rc}$ \\
\hline & $0,10 \mathrm{Rc}$ & 50 & 4 & $0,40 \mathrm{RC}$ \\
\hline
\end{tabular}

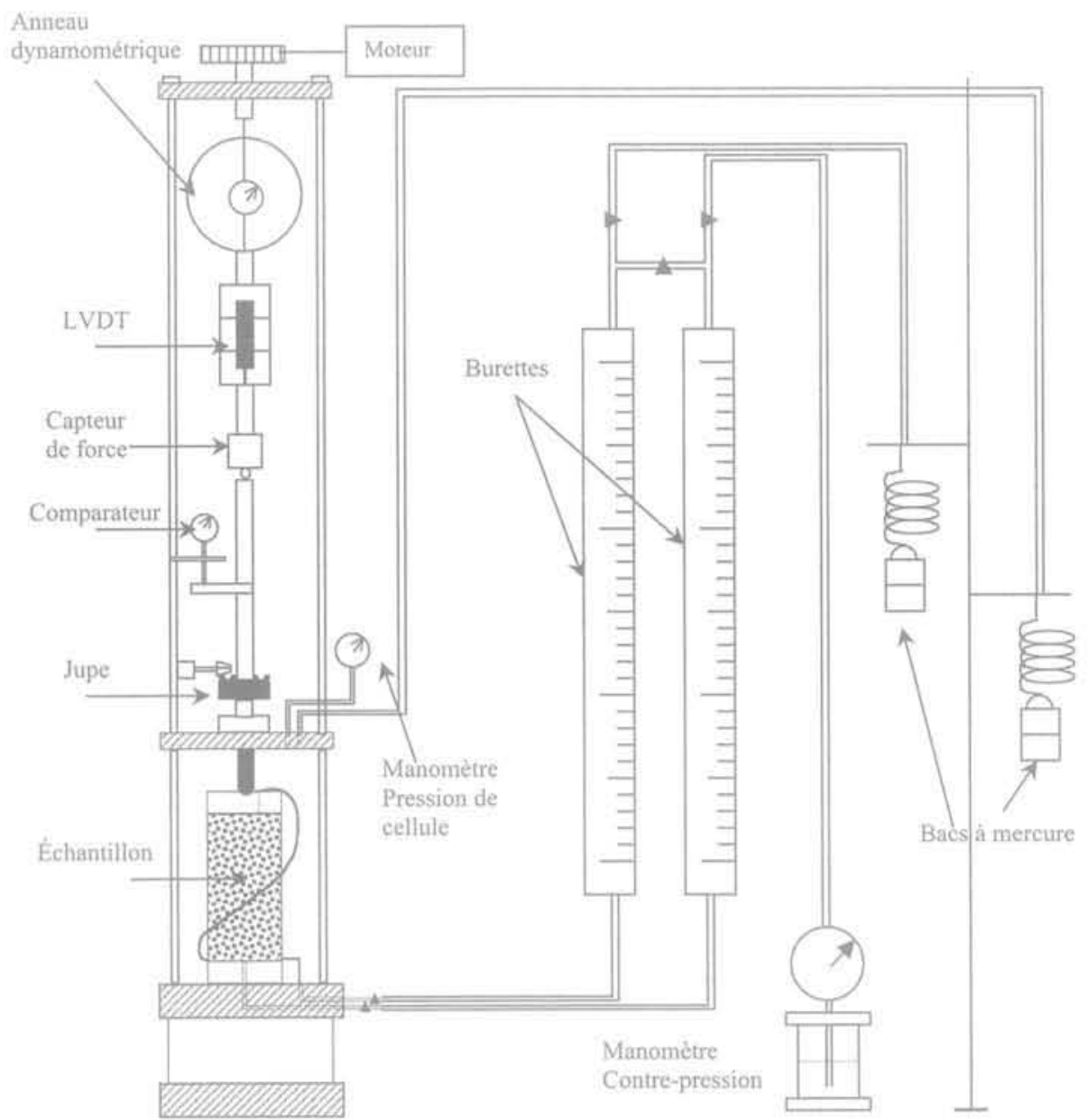

fig.4 Schéma du système triaxial pour les essais de fluage avec confinement.

Schematic representation of the triaxial apparatus for confined compression creep.

TABLEAU III Caractéristiques des essais de fluage sur 100 jours avec confinement. Characteristics of the confined creep tests performed over a period of 100 days.

\begin{tabular}{l|c|c}
\multicolumn{1}{c|}{ Nature des échantillons } & $\begin{array}{c}\text { Méthode de mise en place } \\
\text { du sable }\end{array}$ & $\begin{array}{c}\text { Te chargement } \\
\text { SF }+ \text { gel de silice }\end{array}$ \\
\hline SF + coulis minéral & Pluviation & $0,20 \mathrm{RC}$ \\
\hline SF non traité & Pluviation & $0,70 \mathrm{Rc}$ \\
\hline
\end{tabular}

(*) Pour le sable de Fontainebleau non traité, la résistance de référence est égale au dèviateur maximal sous contrainte de confinement de $0.1 \mathrm{MPa}$. 
TABLEAU IV Caractéristiques des essais de fluage par incréments avec confinement.

Characteristics of the incremental confined creep tests.

\begin{tabular}{|c|c|c|c|c|}
\hline $\begin{array}{c}\text { Nature } \\
\text { des échantillons }\end{array}$ & $\begin{array}{c}\text { Valeur } \\
\text { de l'incrément }\end{array}$ & $\begin{array}{l}\text { Nombre } \\
\text { d'incréments } \\
\text { à la rupture }\end{array}$ & $\begin{array}{l}\text { Taux } \\
\text { de chargement } \\
\text { à la rupture }\end{array}$ & $\begin{array}{l}\text { Durée } \\
\text { de l'essai } \\
\text { (jours) }\end{array}$ \\
\hline SF + gel de silice & $0,05 \mathrm{Rc}$ & 12 & $0,60 \mathrm{Rc}$ & 203 \\
\hline $\mathrm{SF}+$ coulis de ciment & $0,10 \mathrm{Rc}$ & 16 & $(+\infty)$ & 183 \\
\hline $\mathrm{SF}+$ coulis minéral & $0,10 \mathrm{RC}$ & 8 & $0,90 \mathrm{Rc}$ & 109 \\
\hline SF non traité & $0,10 \mathrm{Rc}$ & 8 & $0,80 \mathrm{Rc}$ & 98 \\
\hline
\end{tabular}

(*) Cét échantillon n'est pas allè à la rupture

au paragraphe précédent. Si l'on porte en abscisse les charges et en ordonnée les pentes de fluage correspondantes, les points de la courbe ainsi construite se placent, en général, sur deux branches distinctes et sensiblement rectilignes. L'intersection de ces deux droites définit la charge de fluage (Fig. 6). Après une croissance lente et proportionnelle à la charge, la pente de fluage se met brutalement à augmenter rapidement. Cette croissance rapide correspond à un changement de comportement du matériau. Il est admis que ce changement se produit pour une charge à partir de laquelle la rupture a lieu au bout d'un temps fini, d'où une détermination de la charge limite (Cambefort et Chadeisson, 1961): En fait, l'utilisation de la limite de fluage prend réellement son sens pour les essais réalisés par incréments car elle permet d'extrapoler les résultats au-delà de la durée de l'essai. Pour le fluage de longue durée, la détermination de la charge limite de fluage ne donne guère plus d'informations sur la résistance à long terme que l'essai lui-même.

\section{5}

\section{Analyse des résultats}

\section{1}

\section{Influence de la mise en place du sable}

Deux modes de mise en place du sable, la pluviation et la densification manuelle, ont été comparés sur des essais de fluage en compression simple d'une durée de 100 jours réalisés sur des échantillons de sable de Fontainebleau injecté au gel de silice. La mise en place du sable n'a pas eu d'effet sur la charge à la rupture puisque dans les deux cas, la charge limite de fluage est comprise entre 0,05 et 0,10 Rc (Fig. 7a et b). En revanche, la mise en place du sable a eu un effet considérable sur les valeurs des déformations de fluage. En effet, les pentes de fluage des échantillons dont le sable a été mis en place par pluviation sont 40 fois plus faibles que celles des échantillons mis en place par densification manuelle (Fig. 8), Le sable mis en place par pluviation est légèrement moins dense que le sable mis en place par densification manuelle et pourtant les déformations de fluage sont plus faibles dans le cas du sable préparé par pluviation. Les écarts observés sont dus à des différences au niveau de la matrice du sable injecté. Deux facteurs peuvent être à l'origine de ces écarts, d'une part l'anisotropie de structure due au fait que les grains de sable ne se déposent pas de la même façon selon que le sable a été mis en place par densification manuelle ou par pluviation, d'autre part l'homogénéité, meilleure dans le cas de la pluviation, qui doit favoriser une meilleure stabilité du matériau.

\section{9}

\section{Influence d'un chargement par incréments}

Seule l'influence d'un chargement réalisé par incréments de courte durée a été étudiée par comparaison à un chargement unique de longue durée (100 jours). Les essais ont été réalisés sur du sable de Fontainebleau injecté au gel de silice. Cinq essais de fluage en compression simple ont été réalisés en utilisant des incréments de 0,05 Rc ou 0,10 Rc d'une durée de 15 ou 50 minutes. Les pentes de fluage sont relativement proches, ce qui indique qu'elles ont été peu influencées par l'amplitude et la durée des incréments. En revanche, ces deux paramètres ont joué un rôle sur la valeur de la charge limite de fluage. En effet, la charge limite de fluage augmente lorscue la durée des incréments diminue à incrément d'amplitude constante et inversement lorsque l'amplitude de l'incrément augmente à durée de l'incrément constante (Ribay, 2001) (Fig. 9). La charge limite de fluage pour un chargement par incrément de courte durée est comprise entre 0,30 et 0,40 Rc selon l'amplitude et la durée des incréments tandis qu'elle est comprise entre 0,05 et $0,10 \mathrm{Rc}$ pour les essais de fluage réalisés sur une période de 100 jours (Fig. 7a). Voyiatzoglou (1980) a utilisé cette méthode incrémentale sur les argiles pour déterminer de façon rapide la charge limite de fluage. Mais les essais étaient réalisés en conditions non drainées et donc les conditions de contraintes appliquées aux échantillons étaient différentes de celles appliquées pour cette étude (conditions drainées). L'application d'un chargement incrémental de courte durée conduit à une surestimation de la charge limite de fluage pour le sable injecté dans des conditions d'essais drainées. Cette méthode ne peut donc pas être utilisée comme méthode rapide pour déterminer la charge limite de fluage.

\section{3}

\section{Influence du traitement par injection}

Les essais de fluage avec confinement réalisés à la fois sur du sable de Fontainebleau non traité et injecté 


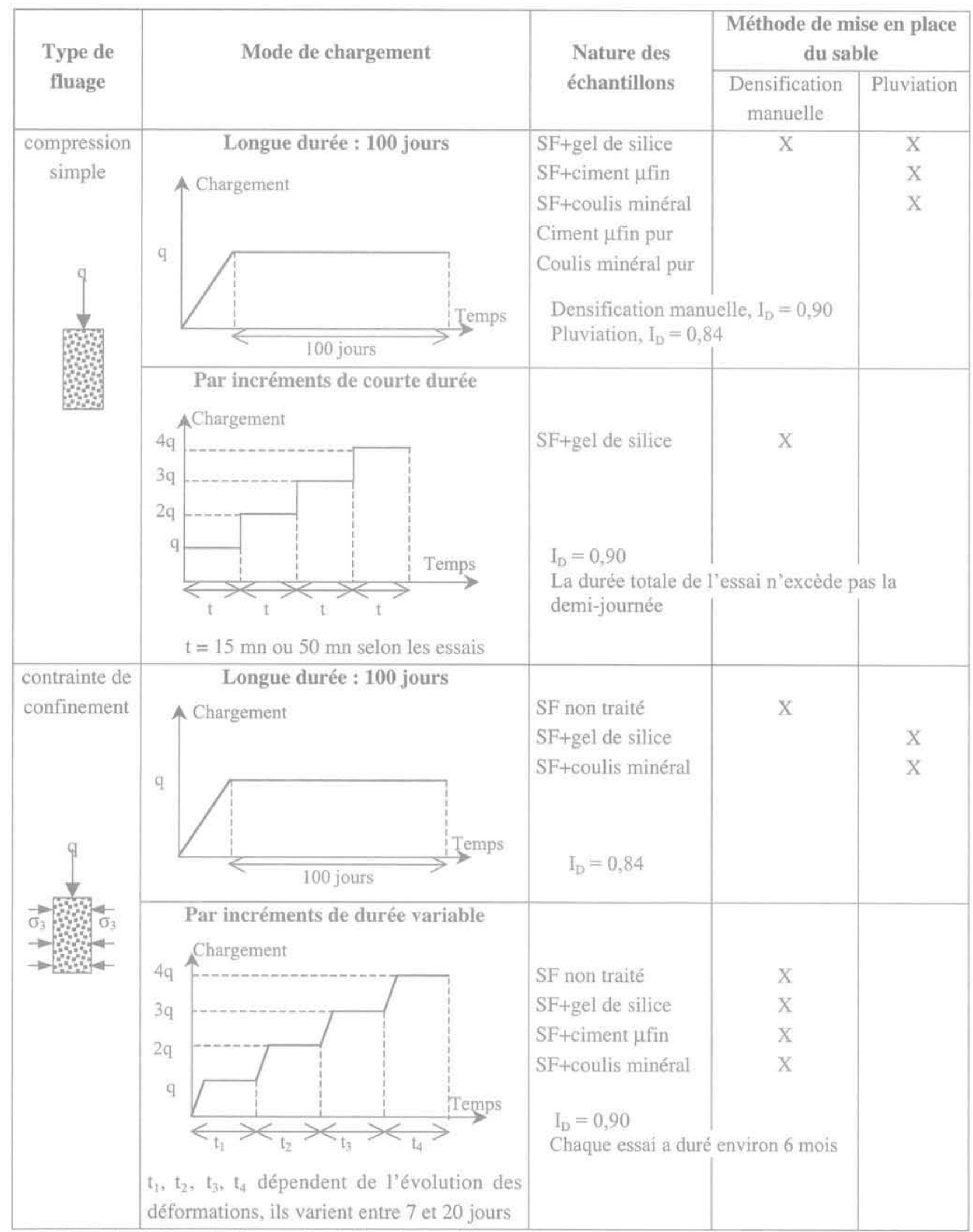

SF : sable de Fontainebleau $-I_{D}$ : Indice de densité

FIG.5 Récapitulatif des essais de fluage réalisés sur le sable non traité, le sable injecté et les coulis purs. Summary of creep tests performed on sand, grouted sand and grouts grouted sand and grouts. 


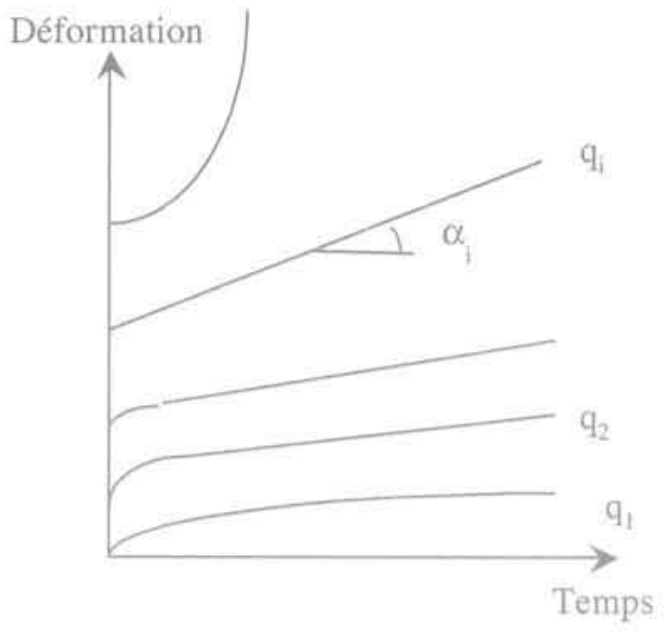

Pente de fluage, $\alpha$

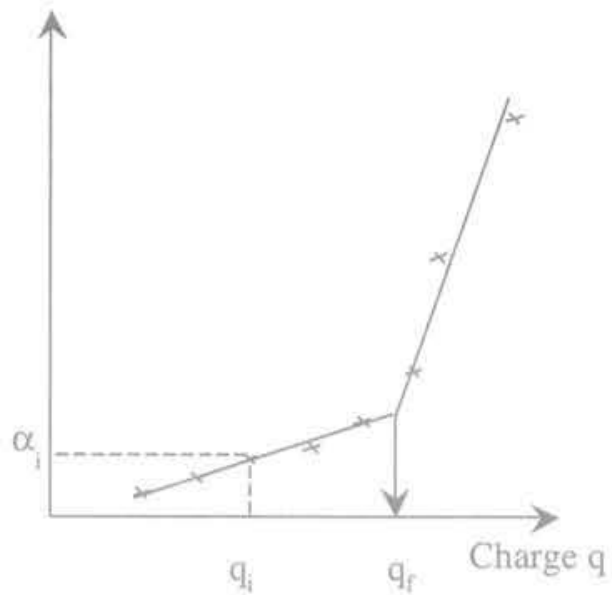

Charge limite de fluage

FiG. 6 Principe de détermination de la charge limite de fluage.

Determination of the creep limit strength.

ont permis de montrer que l'injection a une influence sur le comportement au fluage du sable. En effet pour les essais de fluage réalisés par incréments de longue durée, quel que soit le niveau de chargement et quelle que soit la nature du coulis, la pente de fluage du sable injecté est différente de celle du sable non traité (Fig. 10). De même sur les essais de fluage réalisés sur 100 jours, pour un même niveau de contrainte, le sable injecté au coulis minéral a une pente de fluage inférieure à celle du sable non traité (Fig. 11).

\section{4}

\section{Influence de la nature du coulis}

Sur les essais de fluage en compression simple, les gammes de chargement applicables aux échantillons sont différentes selon la nature du coulis : la charge limite de fluage du sable injecté au gel de silice est comprise entre 0,05 et 0,10 Rc (résistance en compression simple de l'échantillon) tandis qu'elle est supérieure à 0,60 Rc pour le sable injecté au coulis de ciment et au coulis minéral. D'autre part, les pentes de fluage du sable injecté au coulis de ciment micro-fin sont systématiquement plus faibles que celles du sable injecté au coulis minéral (Fig. 12).

Pour les essais de fluage avec confinement réalisés par incréments de longue durée, que les résultats soient exprimés en déformations (Fig. 13) ou en pentes de fluage (Fig. 10), le sable injecté au gel de silice est le matériau qui présente le plus de déformations de fluage et celles-ci sont d'autant plus importantes que la charge augmente. Les déformations du sable injecté au coulis minéral et au coulis de ciment micro-fin sont très faibles et évoluent peu avec le chargement (Ribay et al., 1999),

Tous les résultats, qu'il s'agisse d'essais en compression simple ou avec confinement, montrent que la nature du coulis a une influence considérable sur le comportement au fluage du sable injecté. Le gel de silice va donner au sable un comportement visqueux plus marqué que le coulis de ciment micro-fin et le coulis minéral, qui diminuent les déformations de fluage.

\section{5}

\section{Influence du fluage du coulis pur}

Ata (1993) a montré que le fluage du sable injecté par un gel de silice est fortement influencé par celui du coulis pur. Il a en effet obtenu que le potentiel de fluage (pente $\mathrm{m}$ de Singh et Mitchell (1968)) du sable injecté est pratiquement égal à celui du coulis pur.

Comme pour Ata (1993), les essais de fluage en compression simple d'une durée de 100 jours réalisés ả la fois sur des échantillons de sable injecté et de coulis purs (coulis de ciment et coulis minéral) ont montré que le fluage du sable injecté est influencé par la nature du coulis. Les déformations de fluage du coulis pur sont systématiquement plus élevées que celles du sable injecté à niveau de chargement équivalent, ce qui signifie que le coulis pur est plus visqueux que le sable injecté. Aussi bien pour le coulis de ciment micro-fin (Tableau V) que pour le coulis minéral (Tableau VI), la pente de fluage du coulis pur est systématiquement dix fois plus élevée que celle du sable injecté quel que soit le niveau de chargement (Delfosse-Ribay et al., 2000). Le comportement au fluage du sable injecté semble être le résultat de T'homogénéisation du comportement au fluage du sable non traité (faibles déformations de fluage) et de celui du coulis pur (déformations de fluage plus ou moins importantes selon le coulis). Il semble donc possible de prédire le comportement au fluage du sable injecté à partir de celui du coulis pur et de celui du sable non traité. Du point de vue de l'industriel, cette perspective est très intéressante car s'il est possible de prévoir le comportement du sable injecté en fonction de celui du coulis, il est possible, à l'inverse, de déterminer le coulis à injecter qui conduira aux propriétés recherchées du sable injecté. Cependant, des essais en plus grand nombre et avec un sable différent du sable de Fontainebleau seraient nécessaires pour valider cette hypothèse. En outre, d'autres facteurs tels que l'adhésivité entre le sable et le coulis peuvent également intervenir. De ce fait, la nature du sable et surtout la nature des liaisons entre les grains de sable et le coulis peuvent jouer un rôle sur le comportement final du sable injecté. 
Temps (jours)

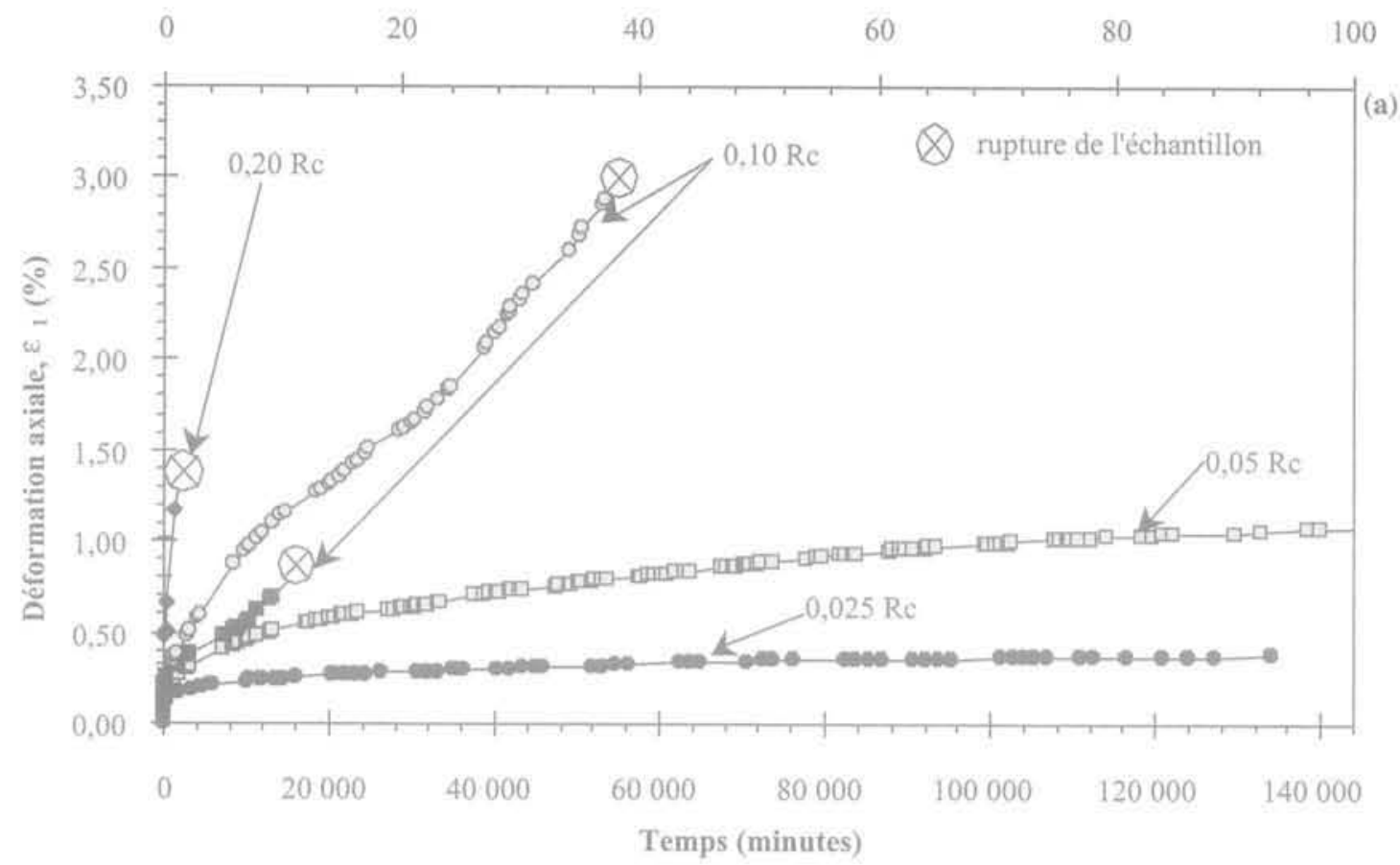

Temps (jours)

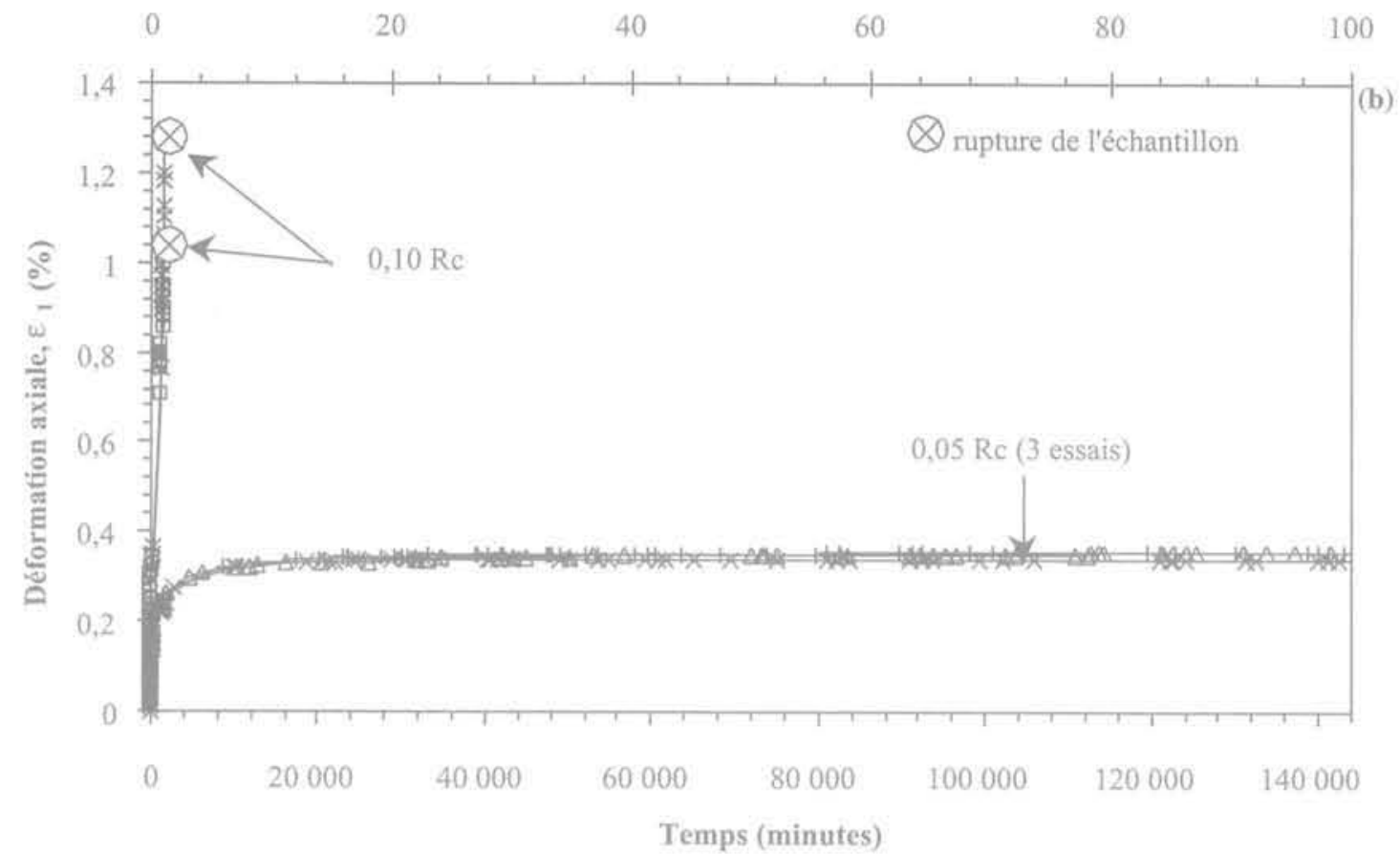

FiG.7 Courbes de fluage - Essai de fluage en compression simple sur 100 jours - sable injecté au gel de silice - Mise en place du sable par: (a) densification manuelle ; (b) pluviation. Creep curves - unconfined compression creep test over a period of 100 days - Silicate grouted sand Method of sand preparation: (a) manual compaction; (b) pluviation.

\section{6}

\section{Influence du confinement}

Lorsqu'un confinement de $100 \mathrm{kPa}$ est appliqué, le sable injecté au gel de silice peut supporter un taux de chargement de 0,20 Ric sans qu'il y ait de rupture sur une période de 100 jours alors qu'en compression simple, un chargement de $0,10 \mathrm{Rc}$ conduit à la rupture,
D'autre part, lorsque le confinement est augmenté de 0 à $100 \mathrm{kPa}$, la pente de fluage du sable injecté au gel de silice passe de $2.10^{-3} \% / \mathrm{mn}$ à $3.10^{-6} \% / \mathrm{mn}$ pour un niveau de chargement équivalent, c'est-à-dire une pente de fluage 6,5 fois plus faible (Fig. 14a). Ces résultats sont en accord avec ceux de Littlejohn et Mollamahmutoglu (1994) sur du sable de Leighton Buzzard injecté par un gel de silice. Les mêmes observations ont été faites sur le sable injecté au coulis minéral, pour 


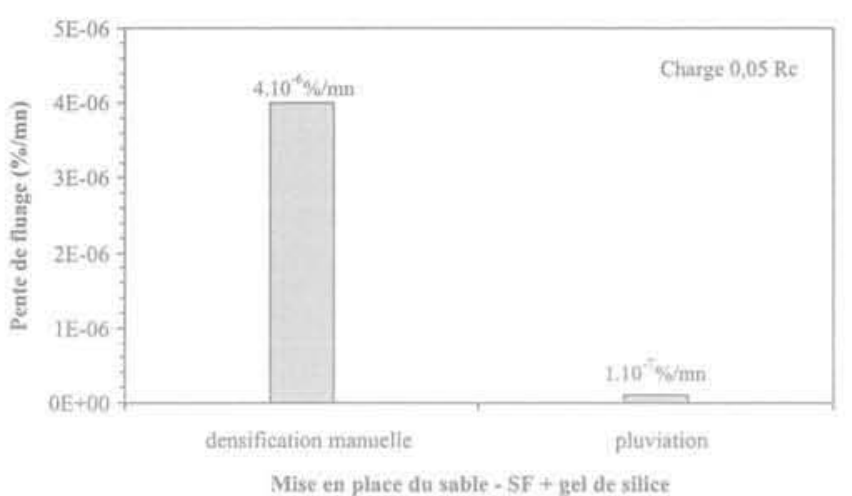

FIG. 8 Influence de la mise en place du sable sur le fluage - Essai de fluage en compression simple sur 100 jours.

Effect of the sand preparation on creep Unconfined creep test over a period of 100 days.

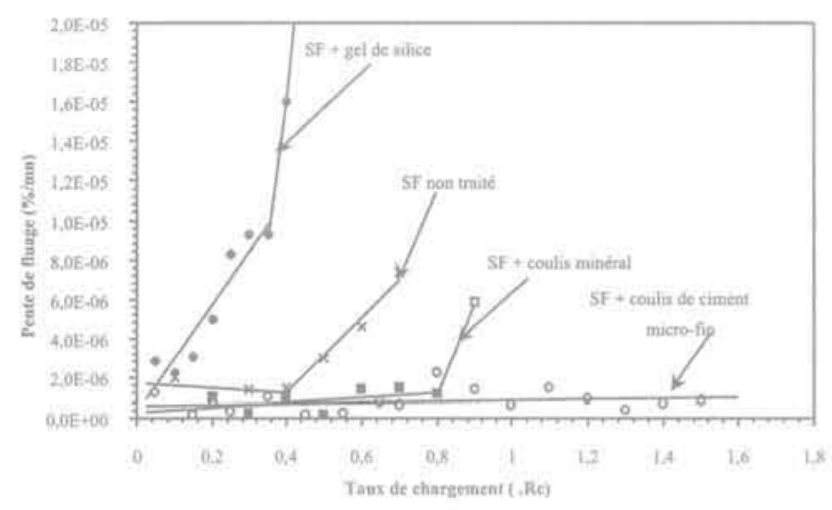

FIG. 10 Influence du traitement par injection et de la nature du coulis - Essai de fluage avec confinement par incréments.

Effect of the grouting treatment and the type of grout - Incremental confined creep test.

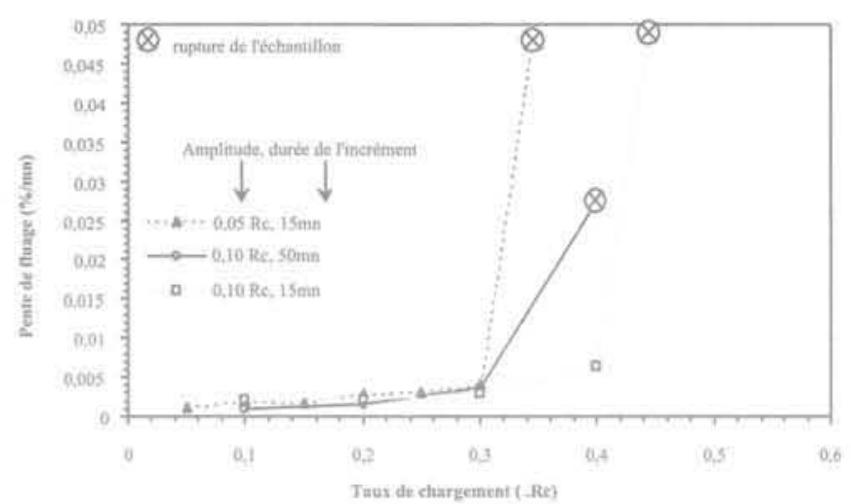

FG.9 Évolution des pentes de fluage avec la charge - Essais de fluage en compression simple par incréments - Sable de Fontainebleau injecté au gel de silice.

Evolution of the creep slope with load - Incremental unconfined compression creep test - Fontainebleau sand grouted with silicate grout.

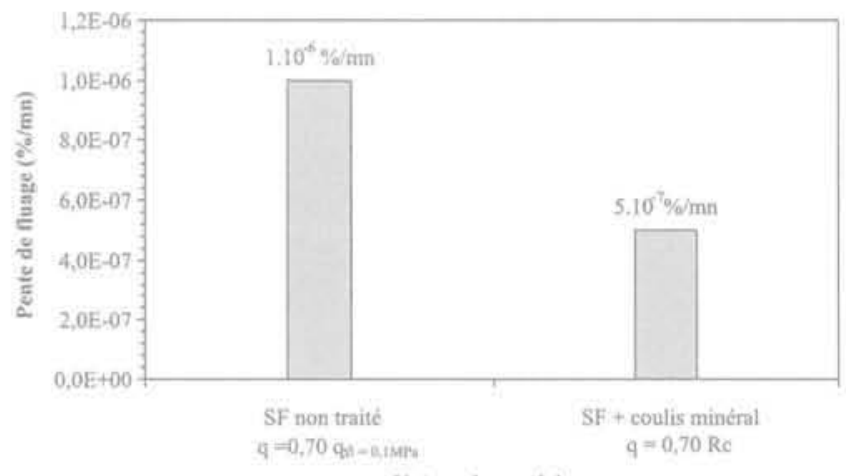

Nature du matériau

FG.11 Influence du traitement par injection sur le fluage - Essai de fluage sur 100 jours avec confinement, $\sigma_{3}=0,1 \mathrm{MPa}$.

Effect of the grouting treatment on creep Confined creep test over a period od 100 days, $\sigma_{3}=0.1 \mathrm{MPa}$.

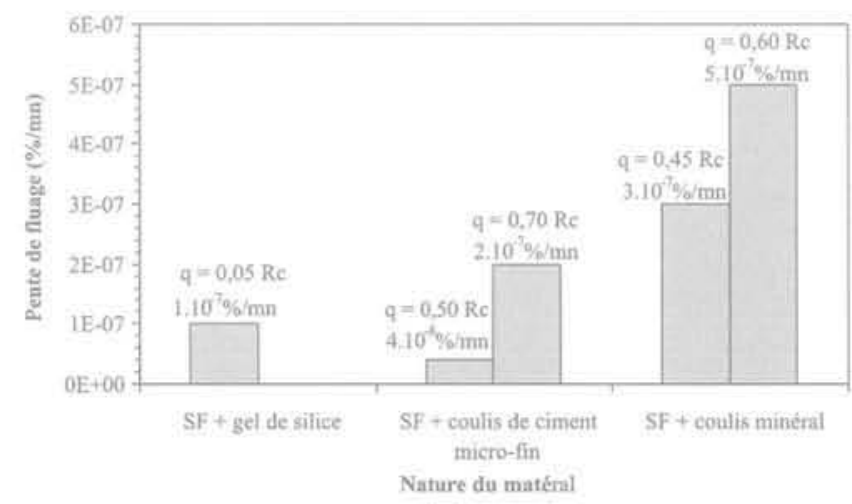

FIG. 12 Influence de la nature du coulis sur le fluage du sable injecté - Essai de fluage en compression simple sur 100 jours.

Effect of the type of grout on grouted sand creep - Unconfined creep test over a period of 100 days. 
Temps (jours)

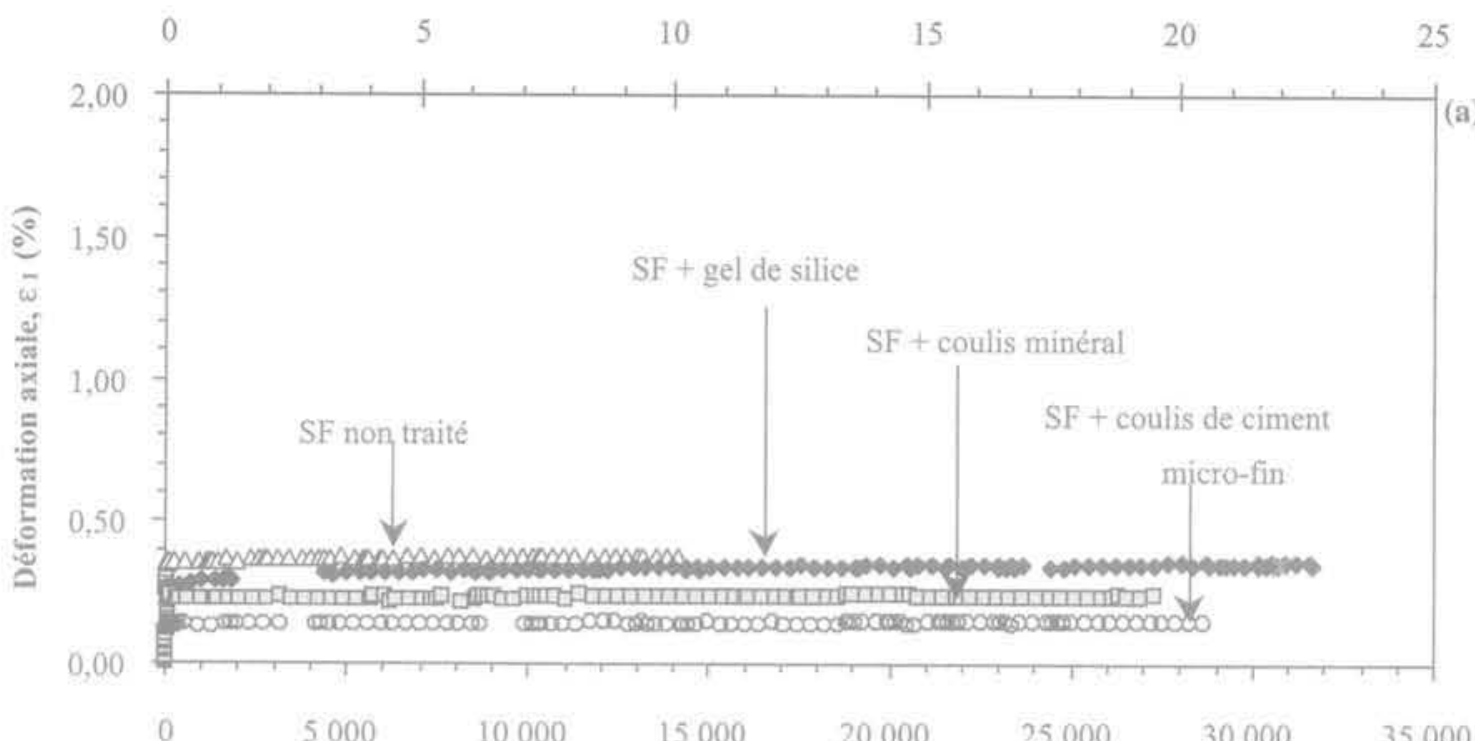

Temps (minutes)

Temps (jours)
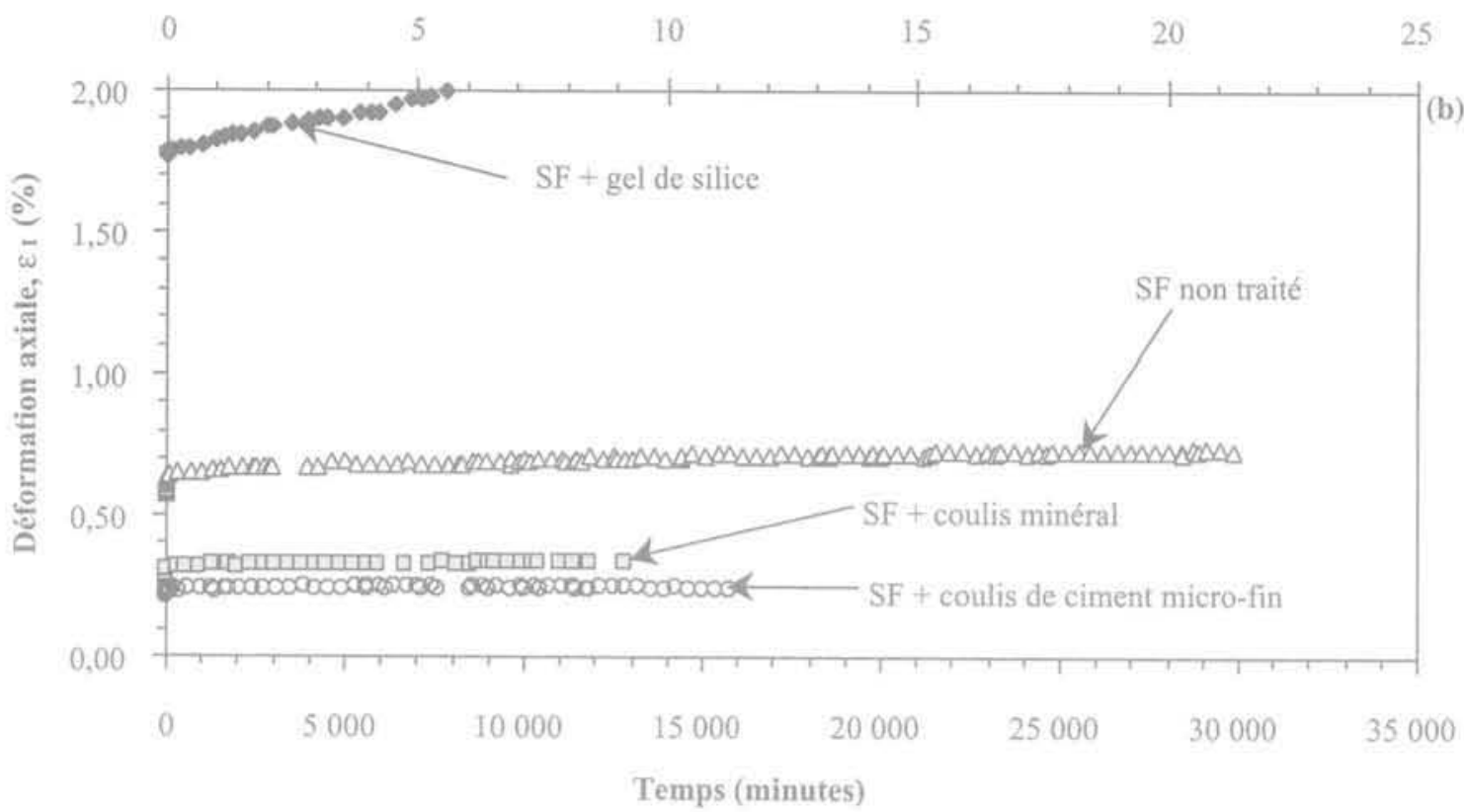

FIG. 13 Essais de fluage avec confinement par incréments:

(a) charge: $20 \%$ de la résistance des échantillons sous confinement de $0,1 \mathrm{MPa}$;

(b) charge : $50 \%$ de la résistance des échantillons sous confinement de $0,1 \mathrm{MPa}$

Incremental confined creep test.

lequel un chargement 1,4 fois plus important a pu être appliqué, en augmentant le confinement de 0 à $100 \mathrm{kPa}$, pour obtenir la même pente de fluage (Fig. 14b). Ces résultats montrent qu'un confinement même faible réduit considérablement les déformations de fluage du sable injecté quelle que soit la nature du coulis.

\section{6}

\section{Conclusion}

Le comportement au fluage du sable de Fontainebleau injecté au gel de silice, chimiquement instable est comparé au comportement au fluage du même sable injecté par deux coulis de nouvelle génération chimiquement stables, un coulis de ciment microfin et un coulis minéral. Le comportement au fluage est caractérisé par des essais en compression simple et des essais avec un confinement réalisés sur des périodes allant de 100 à 200 jours. Il a été réalisé 34 essais de fluage en compression simple sur 100 jours, 5 essais de fluage en compression simple par incréments, 3 essais de fluage avec confinement sur 100 jours, et 4 essais de fluage par incréments de longue durée avec confinement. Ces essais représentent au total 100 niveaux de chargements testés. Les résultats ont été analysés en termes de pente de fluage et de charge limite de fluage. L'analyse des résultats a permis de mettre en évidence 
TABLEAUV Pente de fluage $(\% / \mathrm{mn})$ - Essais de fluage en compression simple sur 100 jours. Creep slope - Unconfined compression creep tests over a period of 100 days.

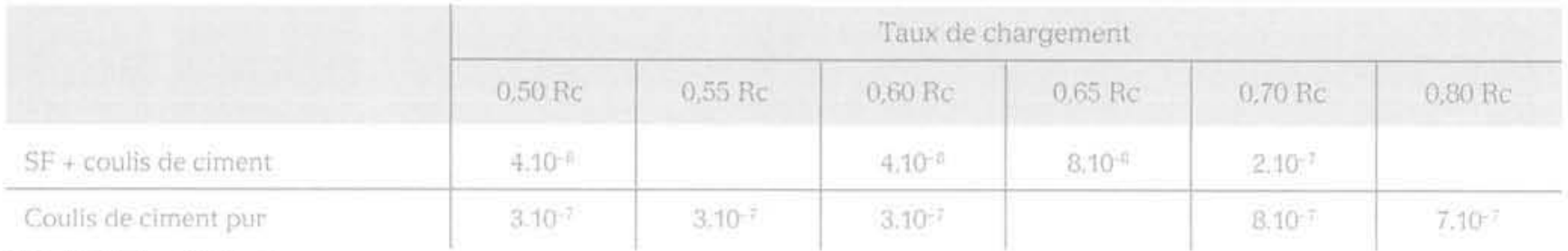

SF: sable de Fontainebleau

TABtEAUV Pente de fluage $(\% / \mathrm{mn})$ - Essais de fluage en compression simple sur 100 jours. Creep slope - Unconfined compression creep tests over a period of 100 davs.

\begin{tabular}{|c|c|c|c|c|c|}
\hline & \multicolumn{5}{|c|}{ Taux de chargement } \\
\hline & $0,35 \mathrm{Rc}$ & $0,40 \mathrm{Re}$ & $0,45 \mathrm{Rc}$ & $0,50 \mathrm{Rc}$ & $0,60 \mathrm{Rc}$ \\
\hline $\mathrm{SF}+$ coulis minéral & & & $5.10^{-2}$ & $5.10^{+2}$ & $3.10^{-7}$ \\
\hline Coulis minéral pur & $6,10^{-7}$ & $3.10^{-6}$ & & $4.10^{-4 i}$ & $6.10^{-6}$ \\
\hline
\end{tabular}
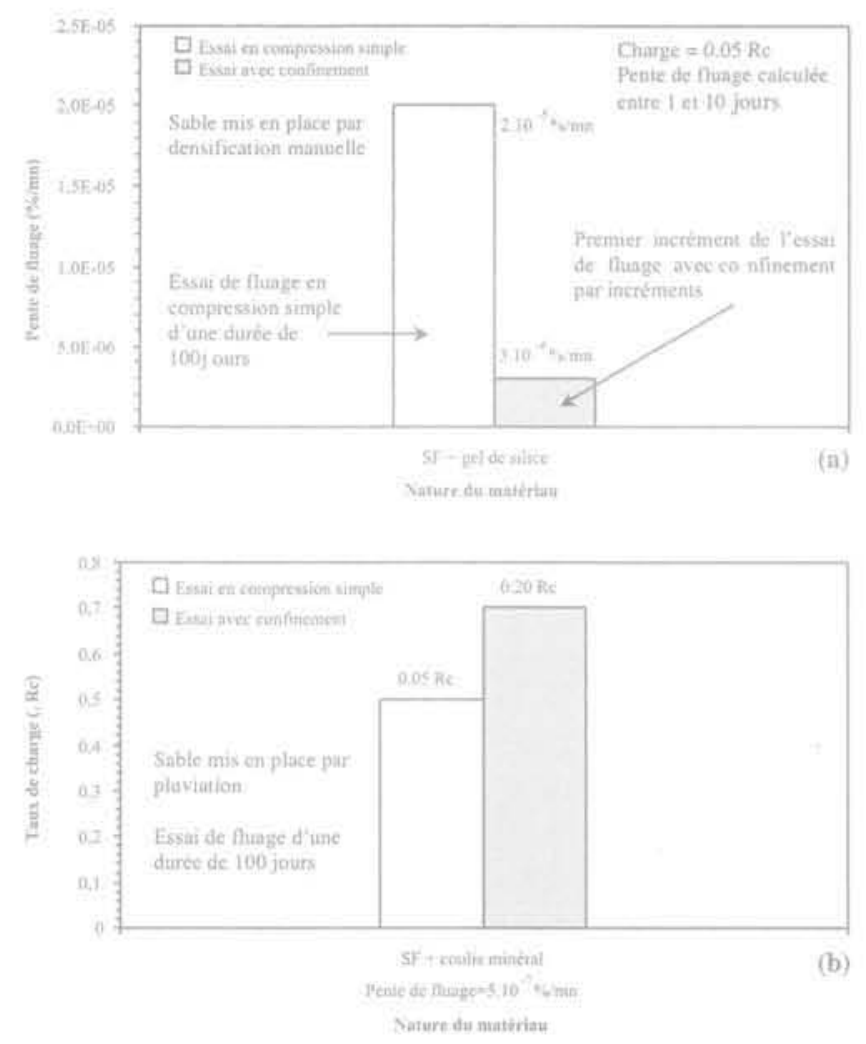

FG, 14 Influence du confinement sur le fluage du sable injecté: (a) sur la pente de fluage à taux de chargement constant; (b) sur le taux de chargement à pente de fluage constante

Effect of the confining pressure on grouted sand creep. le rôle du traitement par injection, et particulièrement l'influence de la nature du coulis. D'autres facteurs, tels que la méthode de mise en place du sable ou la présence d'un confinement ont également été abordés. Les principales conclusions sont les suivantes:

(a) le mode de mise en place du sable a une influence considérable sur le fluage du sable injecté : en effet les échantillons de sable injecté au gel de silice dont le sable a été mis en place par densification manuelle présentent des déformations de fluace 40 fois supérieures à celles des échantillons dont le sable a été mis en place par pluviation:

(b) sur des chargements de courte durée, un chargement par incrément conduit à une surestimation de la charge limite de fluage :

(c) la présence d'un confinement réduit considérablement les déformations de fluage du sable injecté:

(d) l'injection modifie le comportement au fluage du sable. L'injection au gel de silice conduit à un sable injecté très viscueux avec une charge limite de fluage en compression simple comprise entre 0,05 et 0,10 Rc (résistance en compression simple des échantillons). L'injection au coulis de ciment micro-fin et au coulis minéral conduit à des matériaux injectés stables avec des charges limites de fluage superieures à $0,60 \mathrm{Rc}$ :

(e) le coulis de ciment micro-fin pur et le coulis minéral pur présentent des déformations de fluage supérieures à celles du sable injecté. La pente de fluage du coulis pur est systématiquement 10 fois supérieure à celle du sable injecté. Compte tenu de cette propriété, il semble possible de pouvoir caractériser le fluage du sable injecté à partir de celui du coulis pur et du sable pur, Le coulis pur est un matériau visqueux (plus ou moins selon la nature du coulis) et la matrice granulaire agit comme une sorte de "frein » quí limite les défor- 
mations de fluage du coulis qui conduit à un sable injecté systématiquement moins visqueux que le coulis pur.

\section{REMERCIEMENTS}

Los auteurs tiennent à remercier d'the part le laboratoire du CERMES (Centre d'étudies et de recherche en mécanicues des sols) de IÉcole nationale des ponts et chaussées pour sa collaboration dans la fabrication des échantillons par la méthode de pluviation et d'autre part la RATP qui a financé une partie des essais présentés dans cet article.

\section{Bibliographie}

AFTES - 4 Recommandations relatives aux travaux d"iniection pour ouvracies souterrains :. Tunnels et ouvraces souterrains, mai 1988, numéro spécial.

Ata A. - Effect of cohesive and adhesive properties of grouts on the static and the cvelic behavior of qrouted sand. Dissertation. University of Houston. Decembet 1993,320 p.

Borden F.H, Krizek J., Baker W.H. “Creep behavior of silicate grouted sand $n$. Proceedings of the conference on Grouting in Geotechnical Engineering. New Orleans, Louisiana, February 1982 p. $450-469$.

Cambefort H. - Injection des sols ; principe et méthodes (tome 1). Ed. Eyrolles, 1967. $396 \mathrm{p}$.

Cambefort H., Chadideisson R. - a Critere pour l'évaluation de la force portante d'un pieu 3. Proceedings of the Vth ICSMFE, Paris, 1961, p. 23-26

Christopher B.R., Atmazidis D.K.. Krizek A.J. - u Laboratory testing of chemically grouted sand x. Geotechnical Testing Journal, val. 12, n². June 1989, p. 109. 118

Cresswell A.. Barton M.E.. Brown R. "Detemining the maximum density of sands by pluviation $\pi$. Geotechnical Testing Journal, vol. 22, $n^{\circ}$ 4. December 1999, p. 324-328.

Delfosse-Ribay E., Dieran-Maigre I. Cabrillac F., Gouvenot D. - " Influence de-la nature du coulis dinjection sur les proprièrés visquetises des sols injec- tẻs n. Colloque AFGC, Génie civil et environnement. Lyon, 27-28 juin 2000.

Gandais M. - " Tirants définitifs et essais de tirants 1 . Annales de ITTBTP, Janvier, $n^{2}$ 346. Sèrie " sols et fondations " n० 136. 1977. P. 45-67

Gouvenot D, - "State of the Art in European grouting D. Ground improvement vol. 2,1998 , p. 51-67

Gouvenot D. - * Injection des sols et législation relative aux nappes phréatiques 1. Comptes rendus des journées d'études internationales de l'AFTES. Bordeaux, 21-23 octobre 1987, p. 455-459.

Krizek R.I. Michel D.F. Helal M. Borden R.H. Engineering properties of acrylate polymer grout. Conference on groutinc. soil improvement, and geosynthetics. ASCE. New Orleans, 1982, p. 712-724.

Lacour J. Delmas F., Bustamante M. Essais de tirants d'ancrage scellés dans une argile plastique Annales de l'Institut Technique du Batiment et des Travaux Publics, fuin, $n^{\circ} 362$, Série : sols et fordations $n^{2} 157,1978, p .73-91$.

Littlejohn G.S.. Mollamahmutoglu M. Time-dependent behaviour of silicate arouted sand. Grouting in the ground. Thomas Telford, 1994. p. 37-50.

Luong M.P., Gandais M., Allemand P. Compontement mecanique des sols Injectés aux produits chimiques. Annales de ITTBTP, octobre, supplément au n" 3.54, " Sols et Fondations v. $n^{2}=145,1977,0.13-32$.
Mollamahmutogiu M. - Creep behaviour of silicate-ester arouted sand. Ph.D. Thesis, University of Bradford, 1992, 284 p.

Murayama S., Shibata T. - a Flow and stress relaxation of clays $\gg$. Symposium IUTAM. Grenoble. 1964, p. 99-129

Ribay E., Cabrillac R., Gouvenot D. - "Triaxial creep behaviour of grouted sand $x$. International congress, creating with concrete, Dundee. Scotland, 6-10 September 1999 , p. 31-40.

Ribay Delfosse E. - Etude du comportement au fluage et sous-chargement cycligue du sable injecté. Thèse cie Doc. torat, Université de Cergy-Pontoise, 5 juillet 2001, 272 p.

Singh A., Mitchell J.K. - w General stressstrain-time function for soils is Journal of the Soil Mechanics and Foundations division. Proceedings of the ASCE. vol.94, n SM1, January 1968, p.21.46.

Tailliez S. - Etude expérimentale du comportement mécanique des sols granulaires injectés. Thèse de doctorat, Ecole centrale de Paris. 1998, 244 p.

Tan T:- nDiscussion w. Proceedings of the Vth ICSMFE, Paris, vol. 3, 1961, p. 141-143.

VTaloy S.S. Skiblisky A.M. - a Problems of the rheology of soils n. Proceedings of the Vth ICSMFE, vol. 1, Paris, 1961. p. $387-391$.

Voyiatzoglou C. - Contribution à l'êtude de la fatigue et da fluage des argiles saturées. Thèse de doctorat. Ecole centrale de Paris, 30 juin 1980, 184 p. 